\title{
Integrated Photonic Chip Enabled Simultaneous Multi-Channel Wideband Radio Frequency Spectrum Analyzer
}

\author{
Ming Ma, Student Member, IEEE, Rhys Adams, and Lawrence R. Chen, Senior Member, IEEE, \\ Fellow, OSA
}

\begin{abstract}
Radio frequency spectrum analysis is an essential technique for monitoring signal quality in ultra-high-speed optical transmission, broad bandwidth photonic RF arbitrary waveform generation, and for characterization of high repetition rate optical pulse sources. Whereas the intrinsic bandwidth of electronics limits its use for RF spectrum measurement of highspeed signals, a photonic approach based on ultra-fast Kerr nonlinear effects can readily measure the RF spectrum of optical signals having a bandwidth beyond $1 \mathrm{THz}$. The majority of fiberbased or integrated photonic RF spectrum analyzers reported to date can monitor only one channel at a time. We demonstrate how to harness mode-selective excitation of nonlinear optical effects to perform simultaneous multi-channel RF spectrum analysis using a single integrated silicon photonic device. This approach, which can be scaled to a higher number of channels, opens up a new degree of freedom for realizing multi-channel signal characterization using a single integrated photonic device.
\end{abstract}

Index Terms-Microwave photonics, silicon photonics, integrated optics, photonic integrated circuits, microwave signal processing

\section{INTRODUCTION}

$\mathrm{R}$ ADIO frequency spectrum analysis (RFSA) is broadly used in optical signal impairment monitoring [1], broad bandwidth photonic RF arbitrary waveform generation [2], and spectrum characterization of high repetition rate optical pulse sources [3, 4]. The RF spectrum of an optical signal is typically obtained by first using a photodetector for optical-toelectrical conversion followed by measurement using an electrical spectrum analyzer. Restricted by the intrinsic bandwidth of electronics, this technique is not able to measure optical signals having a bandwidth higher than $100 \mathrm{GHz}$. While a trade-off between measurement bandwidth and resolution has to be made, photonic implementation of RFSA by utilizing ultra-fast nonlinear Kerr effects can readily process optical signals with a bandwidth of beyond $100 \mathrm{GHz}$. For instance, it has been reported that photonic RFSA in a highly nonlinear fiber has a 3-dB bandwidth of up to $800 \mathrm{GHz}$ [5]. Moreover, photonic RFSA for a $640 \mathrm{GHz}$ mode-locked laser was also demonstrated [3]. But these implementations in fibers are usually bulky. On the other hand, integrated solutions are more favored since they can greatly reduce device footprint. To date, RFSA has been reported in passive silicon waveguides [6,7], doped silica waveguides [8], and chalcogenide waveguides [9, 10], all of which implement single-channel operation. Multi-channel simultaneous RFSA operation in a single integrated device is more useful because this scheme helps reduce device complexity when compared to simply multiplying single-mode nonlinear waveguides. Although bi-directional propagation and orthogonal polarization in a waveguide can help increase the number of optical signals being characterized, scaling to a higher number of signals is limited in these methods.

Similar to multi-mode fibers [11-13], integrated waveguides can be engineered to support a few propagating spatial modes, which we can make use of to realize parallel (simultaneous) multi-channel all-optical signal processing (i.e., spatial modes are used rather than dedicating one nonlinear waveguide per signal being processed). For example, by harnessing nonlinear optical effects in a mode-selective manner, Ding et al. have demonstrated simultaneous wavelength conversion of two wavelength channels using four wave mixing [14] while we achieved simultaneous regenerative wavelength conversion of two wavelength channels using cross-phase modulation (XPM) [15]. In this paper, we extend this approach to realize multi-channel photonic RFSA. We measure the RF spectra of $640 \mathrm{GHz}$ and $160 \mathrm{GHz}$ optical waveforms simultaneously using uni-directional propagation in a single multi-mode waveguide.
This work was supported in part by the Natural Sciences and Engineering Research Council of Canada (NSERC) CREATE NGON, NSERC CREATE Si-EPIC, and the Fonds de Recherche du Québec - Nature et Technologies (FRQNT).

M. Ma and L. R. Chen are with the Department of Electrical and Computer Engineering, McGill University, 3480 University Street, Montréal, QC, H3A 0E9, Canada (e-mail: ming.ma@mail.mcgill.ca; lawrence.chen@mcgill.ca).

R. Adams is with the Department of Physics, Vanier College, 821 SainteCroix, Montréal, QC, H4L 3X9, Canada (e-mail: adamsr@vanier.college). 


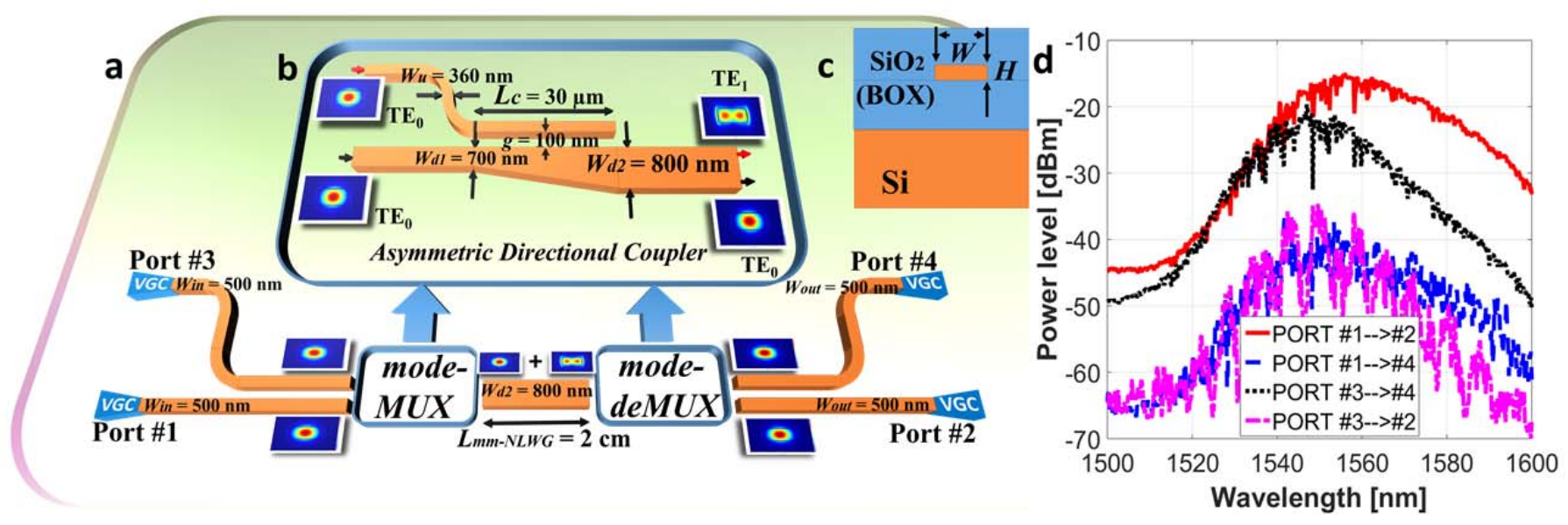

Fig. 1. (a) Schematic of MSND comprising VGCs, m-MUX/m-deMUX, and mm-NLWG. (b) ADC structure. (c) Cross-section of SOI wafer. (d) Intra-channel and inter-channel transmittance (i.e., linear inter-channel cross-talk) over the wavelength span from 1500 to $1600 \mathrm{~nm}$.

\section{INTEGRATED MODE-SELECTIVE NONLINEAR DEVICE}

Fig. 1(a) illustrates the schematic of our integrated device in silicon-on-insulator (SOI), referred to hereafter as the modeselective nonlinear device (MSND). Details of the MSND are given in [15]; we provide here some of the key design and operating characteristics. The MSND is based on silicon waveguides which have a thickness $H=220 \mathrm{~nm}$ and sit on top of a $3 \mu \mathrm{m}$ buried oxide (BOX) layer with a $2 \mu \mathrm{m}$ thick indexmatched top oxide cladding, see Fig. 1(c). The MSND comprises of the following components: vertical grating couplers (VGCs), a mode multiplexer and demultiplexer (mMUX/m-deMUX), and a multi-mode nonlinear waveguide (mm-NLWG) with a length of $2 \mathrm{~cm}$.

The m-MUX and m-deMUX are each based on an asymmetric directional coupler (ADC) as shown in Fig. 1(b). The principle of modal conversion in the ADC is explained in [16]. Briefly, if we launch a light wave at Port \#3 and couple it into the upper waveguide on the input side of the ADC, it will be converted to the $\mathrm{TE}_{1}$ mode on the output side. It then propagates in the $\mathrm{TE}_{1}$ mode of the mm-NLWG; on the other hand, the light wave launched in the lower waveguide on the input side of the ADC (i.e., via Port \#1) will maintain its mode profile at the $\mathrm{ADC}$ output and then travels in the $\mathrm{TE}_{0}$ mode of mm-NLWG. In design, accordingly, the width of the upper waveguide in the ADC is fixed at $360 \mathrm{~nm}$ while that of the lower waveguide extends linearly from $700 \mathrm{~nm}$ to $800 \mathrm{~nm}$ so as to fulfill the phase-matching condition for $\mathrm{TE}_{0}$ to $\mathrm{TE}_{1}$ modal transformation. Furthermore, the coupling gap and length of the ADCs are chosen as $100 \mathrm{~nm}$ and $30 \mu \mathrm{m}$, respectively, in order to obtain a coupling efficiency of up to $91 \%$ in a range of 1500 to $1600 \mathrm{~nm}$.

Finally, mode-selective nonlinear optical effects occur in the mm-NLWG. With a width of $800 \mathrm{~nm}$, the mm-NLWG allows for propagating the $\mathrm{TE}_{0}$ and $\mathrm{TE}_{1}$ modes as well as the first two transverse magnetic modes (which are not excited due to the VGCs). The simulated dispersion and dispersion slope of the mm-NLWG (calculated using Mode Solver from
Lumerical MODE Solutions) at $1550 \mathrm{~nm}$ for the $\mathrm{TE}_{0}$ mode are $-5.55 \cdot 10^{-4} \mathrm{ps} /(\mathrm{nm} \cdot \mathrm{cm})$ and $1.75 \cdot 10^{-6} \mathrm{ps} /\left(\mathrm{nm}^{2} \cdot \mathrm{cm}\right)$, respectively, while the counterparts for the $\mathrm{TE}_{1}$ mode are $1.56 \cdot 10^{-3} \mathrm{ps} /(\mathrm{nm} \cdot \mathrm{cm})$ and $-1.51 \cdot 10^{-5} \mathrm{ps} /\left(\mathrm{nm}^{2} \cdot \mathrm{cm}\right)$, respectively. Furthermore, the calculated effective areas for the $\mathrm{TE}_{0}$ and $\mathrm{TE}_{1}$ modes are $0.094 \mu \mathrm{m}^{2}$ and $0.122 \mu \mathrm{m}^{2}$, respectively, at $1550 \mathrm{~nm}$; the corresponding nonlinear coefficients are $194 \mathrm{~m}^{-1} \mathrm{~W}^{-1}$ and $150 \mathrm{~m}^{-1} \mathrm{~W}^{-1}$ (using a nonlinear index $n_{2}=4.5 \cdot 10^{-18} \mathrm{~m}^{2} / \mathrm{W}$ [17]). These values are crucial for realizing large bandwidth of the RFSA, as described in Section III. We designate the transmission from Port \#1 to Port \#2 as Channel \#1 and accordingly, the transmission from Port \#3 to Port \#4 as Channel \#2. Fig. 1(d) depicts the measured intra-channel transmissions within the Channel \#1 or \#2 and inter-channel transmission between the Channel \#1 and \#2. These spectral traces take into account both coupling loss ( $\sim 6 \mathrm{~dB} /$ coupling) and propagation loss of the MSDN. As can be observed, the linear inter-channel cross-talk (i.e., either from Port \#1 to \#4 or from Port \#3 to \#2) lies at least $~ 15 \mathrm{~dB}$ below than the corresponding intra-channel scenarios.

The principle of operation for exciting nonlinear optical effects in a mode-selective manner is as follows. If we launch a high-power pump along with a probe at Port \#1, they then propagate in the $\mathrm{TE}_{0}$ mode of the mm-NLWG or equivalently, on Channel \#1 of the MSND; meanwhile, nonlinear interactions (e.g., cross-phase modulation (XPM) and fourwave mixing (FWM)) between them occur in the $\mathrm{TE}_{0}$ mode too. Similarly, the pump and probe that are launched at Port \#3 will travel in the $\mathrm{TE}_{1}$ mode of the mm-NLWG (or on Channel \#2 of the MSND) and accordingly, nonlinearities arise within the $\mathrm{TE}_{1}$ mode of the mm-NLWG. However, no nonlinear phenomena occur if the signals are launched at separate ports because they will propagate in different modes of the mmNLWG and the inter-modal nonlinear interaction is very limited.

The nonlinear cross-talk was characterized by comparing the highest XPM-induced power level around the probe carrier when both probe and pump signals were launched on the same 
channel (e.g., both probe and pump are on Channel \#1) to when they are launched on different channels (e.g., the probe is on Channel \#1 while the pump is on Channel \#2). Detailed results are provided in [15]; in brief, the nonlinear interchannel cross-talk at $1540 \mathrm{~nm}$ and $1560 \mathrm{~nm}$ is $\sim-30 \mathrm{~dB}$. From the nonlinear cross-talk characterization, we estimate the nonlinear coefficients for inter-modal interactions to be $\sim 0.19$ $\mathrm{m}^{-1} \mathrm{~W}^{-1}$ from the $\mathrm{TE}_{1}$ to $\mathrm{TE}_{0}$ modes and $\sim 0.15 \mathrm{~m}^{-1} \mathrm{~W}^{-1}$ from the $\mathrm{TE}_{0}$ to $\mathrm{TE}_{1}$ modes.

\section{RFSA BANDWIDTH MEASUREMENT}

The theoretical derivations for measuring RF spectrum by an optical spectrum analyzer is well developed in [5]. If a signal-under-test (SUT) is co-polarized with a monochromatic light source (in our case, a CW light source) and they both propagate through an optical nonlinear medium, the phase of the $\mathrm{CW}$ light is modulated by the temporal power profile of the SUT because of XPM. By measuring the optical spectrum of the modulated CW light $O(\omega)$, the RF spectrum of the SUT (denoted as $S(\omega)$ ) can be obtained as follows:

$$
O(\omega) \propto|m|^{2} \cdot S\left(\omega-\omega_{0}\right)
$$

where $\omega_{0}$ is the carrier frequency of the $\mathrm{CW}$ light. $m$ is equal to $\left(i \cdot 4 \pi \cdot n_{2} / \lambda-\beta_{T}\right) \cdot L$ in silicon nonlinear waveguides, where $n_{2}$ and $\beta_{T}$ are the Kerr nonlinear coefficient and two-photon absorption (TPA) coefficient, respectively; $L$ is the waveguide length; $\lambda$ is the average wavelength of the SUT. Furthermore, an estimation of the maximum RFSA bandwidth in an arbitrary nonlinear devices is given by [8]:

$$
\Delta f_{\max }=\frac{1}{L\left|2 D \Delta \lambda+\frac{\partial D}{\partial \lambda}\right|_{\lambda_{s}} \cdot \Delta \lambda^{2} \mid}
$$

where $D$ is the dispersion parameter and $\partial D / \partial \lambda$ is the dispersion slope (both at the SUT wavelength); and $\Delta \lambda$ is the wavelength detuning between CW probe and the SUT. Based on the simulated dispersion profiles, the maximum theoretical bandwidths on Channel \#1 and \#2 are calculated $4.5 \mathrm{THz}$ and $1.5 \mathrm{THz}$, respectively, for a $10 \mathrm{~nm}$ detuning in a $2 \mathrm{~cm}$ long waveguide. Notice that this estimation does not take into account high-order dispersions which could reduce the bandwidth potential.

The bandwidth (BW) measurement of the RFSA was performed by combining the beat signal from two $\mathrm{CW}$ signals centered at $\lambda_{\mathrm{s}}=1562.5 \mathrm{~nm}$ with a CW probe at $\lambda_{\mathrm{p}}=1535 \mathrm{~nm}$, as illustrated in Fig. 2 [5]. The choice for $\lambda_{\mathrm{s}}$ and $\lambda_{\mathrm{p}}$ was determined by the spectral response of the VCGs and the availability of optical filters and amplifiers. The beat frequency $(d f)$ was adjusted by tuning the two $\mathrm{CW}$ signals away from $\lambda_{s}$. The bandwidth on each channel power was then determined by measuring the power level of the XPM-induced side-bands at $\pm d f$ (by an OSA with a $0.1 \mathrm{~nm}$ resolution bandwidth) while varying the detuning frequency $d f$. The total power launched onto Channel \#1 is $23.4 \mathrm{~mW}$ (probe alone 8.9

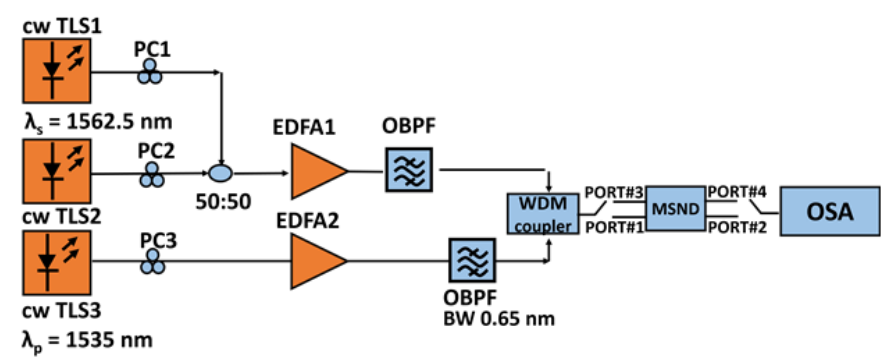

Fig. 2. Experimental setup for RFSA BW measurement. cw: continuouswave; TLS: tunable laser source; PC: polarization controller; EDFA: Erbiumdoped fiber amplifier; OBPF: optical bandpass filter; OSA: optical spectrum analyzer.

$\mathrm{mW}$ ) whereas the counterpart onto Channel \#2 is $37.1 \mathrm{~mW}$ (probe alone $15.1 \mathrm{~mW}$ ), which ensures negligible nonlinear absorption [18]. Figs. 3(a) and 3(b) show the XPM-induced sidebands on Channel \#1 and Channel \#2, respectively, for different values of beat frequency $d f$; the corresponding sideband power as a function of $d f$ is given in Fig. 3(c) [the curves shown are based on fitting a $2^{\text {nd }}$-order polynomial function to the corresponding measured points]. In Fig. 3(c), optical powers at higher frequencies are all normalized to the value at $0.125 \mathrm{THz}$ (i.e., a wavelength detuning between the two $\mathrm{CW}$ signals of $1 \mathrm{~nm}$ ) so as to compare the bandwidths on both channels (since photonic RFSA offers the possibility of characterizing signals with frequencies beyond what might be conventionally achieved with electronics, e.g., beyond $50 \mathrm{GHz}$ (or $0.4 \mathrm{~nm}$ )), we chose to use a $1 \mathrm{~nm}$ detuning step size in the BW measurements with the smallest detuning set at $1 \mathrm{~nm}$. Note that the measurements shown in Fig. 3(c) can be reproduced for the same operating conditions (i.e., source wavelengths and input power levels). The fluctuations arise in large part from ripples in the spectral amplitude response of the VGCs (the typical peak-to-peak ripple for a VGC-to-VCG test structure is $\sim 3.6 \mathrm{~dB}$ ). As can be observed, the BW on Channel \#1 is estimated as $>2.0 \mathrm{THz}$. Similarly, in Fig. 3(b), the 3-dB bandwidth on Channel \#2 is estimated as $\sim 0.5 \mathrm{THz}$. Notice that with the current configuration for $\lambda_{\mathrm{s}}$ and $\lambda_{\mathrm{p}}$, the XPM RF spectral tones will overlap with the four-wave mixing idlers of the two beating $\mathrm{CW}$ signals when the detuning is $\geq 11 \mathrm{~nm}$, thereby restraining the effective BW to $1.4 \mathrm{THz}$. Notice also that on Channel \#1, the side-band power for some larger values of detuning (e.g., between 0.25 and $1.25 \mathrm{THz}$ ) are greater than that at the lowest detuning because the VGC's response has a quasi-parabolic shape and a 3-dB power variation in the frequency range of interest, resulting in amplitude variation in the XPM-induced sidebands. This behavior is not as pronounced for Channel \#2 as it has a smaller bandwidth. We attribute the difference between the calculated and measured BWs to the following. First, the measured BWs take into account all orders of waveguide dispersion whereas the calculated ones only account for the dispersion and dispersion slope. Second, the ripples, bandwidth, and shape of the VGC spectral response will impact the output power of the beat signal and hence the estimation of the bandwidth of the photonic RF spectrum analyzer. 

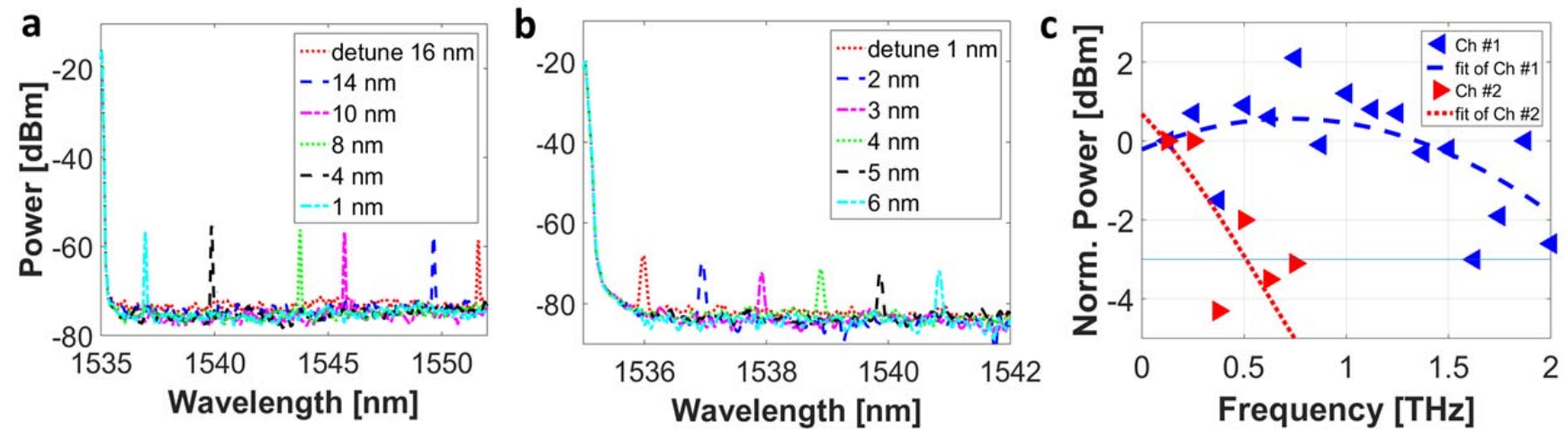

Fig. 3. (a) Optical spectrum evolution as pump detuning increases on Channel \#1, (b) optical spectrum evolution as pump detuning increases on Channel \#2, and (c) corresponding variation in output power of the probe signal as a function of frequency from which the bandwidth of the photonic RF spectrum analyzer is estimated.

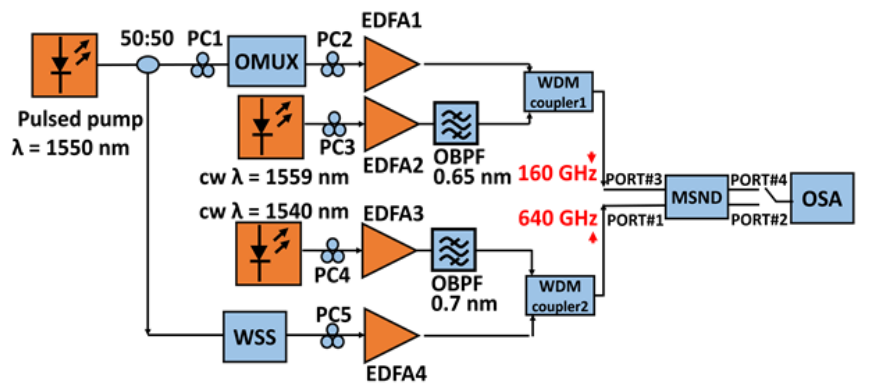

Fig. 4. Experimental setup for multi-channel RFSA with a $640 \mathrm{GHz}$ waveform on Channel \#1 and a pulse train with a $160 \mathrm{GHz}$ repetition rate on Channel \#2. OMUX: optical multiplexer; WSS: Finisar waveshaper.

\section{MUlti-CHANNEL RFSA}

The experimental setup for multi-channel RFSA is shown in Fig. 4, in which we measure a $640 \mathrm{GHz}$ waveform on one channel and a $160 \mathrm{GHz}$ pulse train on the other simultaneously. In detail, a common mode-locked fiber laser at $1550 \mathrm{~nm}$ with a repetition rate of $9.95328 \mathrm{GHz}$ (pump) was divided into two branches. In one branch, an optical multiplexer was used to increase the repetition rate to 160 GHz. The commercial optical multiplexer used is designed to increase the data rate of a $\left(2^{7}-1\right)$ pseudo-random bit sequence (PRBS), i.e., to generate a $40 \mathrm{~Gb} / \mathrm{s}, 80 \mathrm{~Gb} / \mathrm{s}$, or $160 \mathrm{~Gb} / \mathrm{s}\left(2^{7}-\right.$ 1) PRBS from a $10 \mathrm{~Gb} / \mathrm{s}\left(2^{7}-1\right)$ PRBS. As such, it does not perform true pulse repetition rate multiplication. The optical spectrum of the $160 \mathrm{GHz}$ pulse train verifies this as $10 \mathrm{GHz}$ spectral tones are clearly present, as opposed to only comprising spectral tones separated by $160 \mathrm{GHz}$ for the case of true pulse repetition rate multiplication. In the other branch, a $640 \mathrm{GHz}$ sinusoidal waveform was generated by using optical heterodyning (two tones with a $640 \mathrm{GHz}$ frequency detuning were filtered and beat with each other). The probe fed onto Channel \#1 was centered at $1540 \mathrm{~nm}$ and the probe onto Channel \#2 was set at $1559 \mathrm{~nm}$. Both probes and pumps were amplified and combined by WDM couplers and their powers launched into the MSND are the following: probe and pump powers were in $31.6 \mathrm{~mW}$ and $69.2 \mathrm{~mW}$, respectively, on Channel \#1 while the counterparts on Channel \#2 were in 32.3 $\mathrm{mW}$ and in $63.1 \mathrm{~mW}$, respectively. Given these power levels, we estimate the nonlinear phase shift to be $\sim 0.12$ radians for both Channel \#1 and Channel \#2 in order to ensure measurement accuracy [5]. As before, there is negligible nonlinear absorption in the silicon waveguide for these power levels. The spectral measurements on each channel were observed at the output port which corresponds to the probe input port on the same channel. We take advantage of a highresolution OSA (in a $20 \mathrm{MHz}$ resolution) to obtain more details on the optical spectrum and hence photonic RF spectrum. 

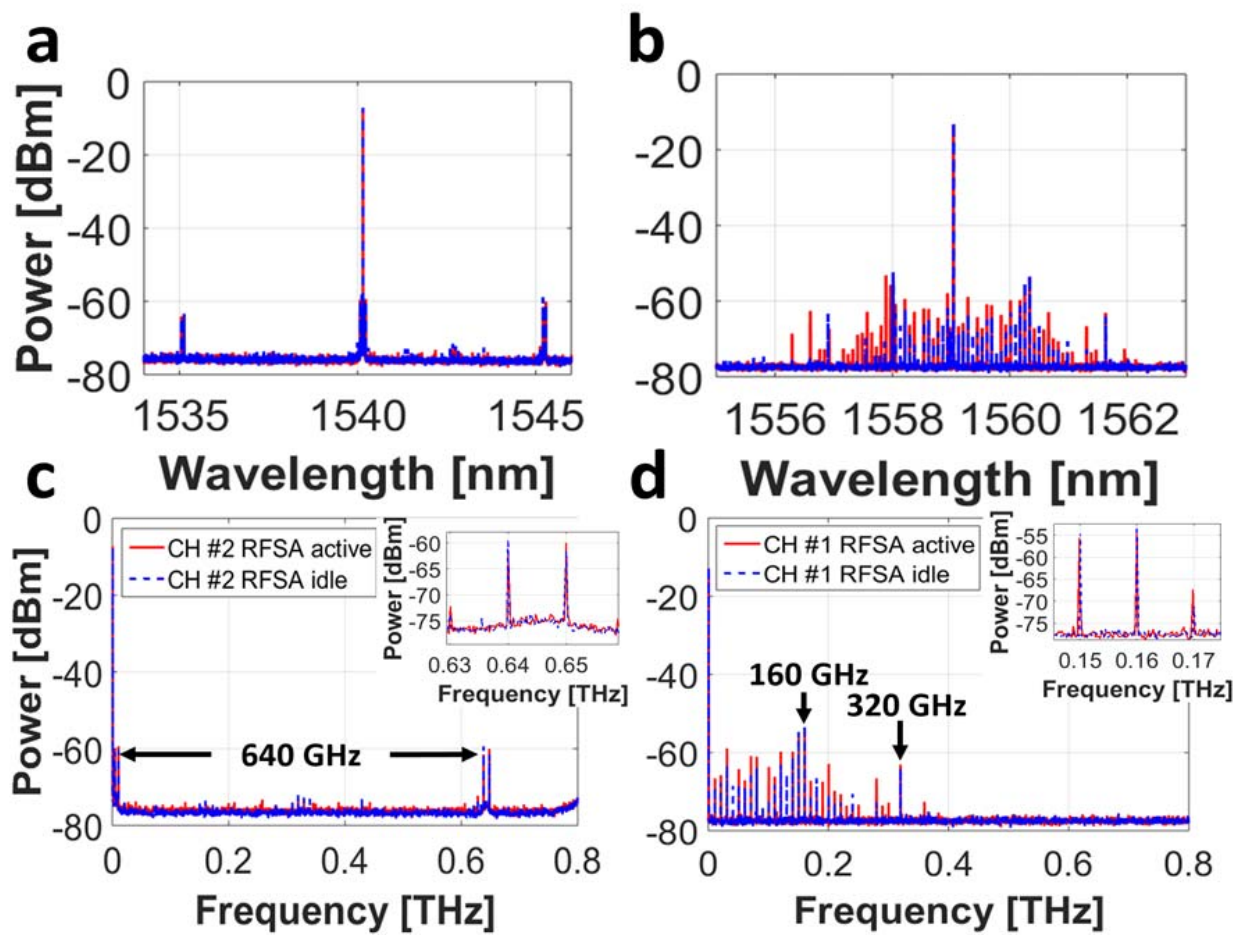

Fig. 5. Performance of simultaneous multi-channel RFSA when the probe carriers are shifted by $19 \mathrm{~nm}$. XPM-induced sidebands are observed on (a) Channel \#1 and (b) Channel \#2. The half sideband RF spectrum on are illustrated in (c) for Channel \#1 and (d) for Channel \#2. Enlarged spectra around the $640 \mathrm{GHz}$ and 160 $\mathrm{GHz}$ tones are shown in the insets. The resolution bandwidth of the OSA is $20 \mathrm{MHz}$.

Figs. 5(a) and (b) show the optical spectra measured at the output of Channel \#1 and the output of Channel \#2 during simultaneous RFSA operation, respectively. In both plots, the red solid curves represent when both channels are active and the blue dashed curves corresponds to when only the observed channel is in operation. Figs. 5(c) and (d) show the corresponding RF spectra (half sideband) on Channel \#1 and Channel \#2. Note that a $640 \mathrm{GHz}$ tone is detected properly on Channel \#1. For Channel \#2, the RF spectrum of the $160 \mathrm{GHz}$ pulse train shows harmonics at $10 \mathrm{GHz}$ and not just tones at $160 \mathrm{GHz}$ as would be the case for true pulse repetition rate multiplication. During the simultaneous RFSA, the $640 \mathrm{GHz}$ tone on Channel \#1 remains unchanged and the $160 \mathrm{GHz}$ spectral tone on Channel \#2 does not manifest itself on Channel \#1. Similarly, the $640 \mathrm{GHz}$ tone from Channel \#1 does not appear in the RFSA on Channel \#2 as cross-talk. These outcomes validate the fact that the RFSA on each channel in our MSND is free from inter-channel interference during the multi-channel simultaneous operation.

Next we characterize further the inter-channel interference in the MSND by positioning the probes with $1 \mathrm{~nm}$ wavelength offset between the two channels. In the measurements as shown in Fig. 6, we maintained the Channel \#1 configuration, i.e., probe at $1540 \mathrm{~nm}$, whereas the probe on Channel \#2 was moved to $1541 \mathrm{~nm}$. During an active Channel \#2, the $640 \mathrm{GHz}$ tone on the Channel \#1 remained the same while the tones from Channel \#2 were not found (see Fig. 6(c)). More specifically, if we focus on the RF tone at $640 \mathrm{GHz}$ in Channel $\# 1$, the power of this tone is within $0.1 \mathrm{~dB}$ when Channel \#2 (with the $160 \mathrm{GHz}$ pulse train) is active or not. Similarly, the power of the $160 \mathrm{GHz} \mathrm{RF}$ tone in Channel $\# 2$ is within $1 \mathrm{~dB}$ when Channel \#1 (with the $640 \mathrm{GHz}$ pulse train) is active or not. Another difference that occurred to Channel \#1 was the coupled $1541 \mathrm{~nm}$ carrier from Channel \#2 via the linear interchannel cross-talk. The inter-channel linear cross-talk from Channel $\# 1$ to $\# 2$ at $1540 / 41 \mathrm{~nm}$ is $\sim-25 \mathrm{~dB}$ and the corresponding one from Channel $\# 2$ to $\# 1$ is $\sim-22 \mathrm{~dB}$ (see Fig. $1(d))$. The presence of the carrier is clearly unwanted and in some applications, and reducing the linear cross-talk further will improve overall performance. Nevertheless, in our results, apart from the leaked carrier, the RF spectra of the signals under test are not impacted significantly.

As a further demonstration, we use the photonic RFSA for monitoring signal impairment (single channel operation). In particular, we measure the RF spectrum of the $160 \mathrm{GHz}$ pulse train after propagation through various lengths of standard single mode fiber (SMF) to induce different levels of dispersion (the length of SMF was placed after the modelocked fiber laser and before the OMUX). Since the repetition rate of the input pulse train to be characterized is known, we can focus on a specific RF tone as obtained from the XPMinduced optical spectrum. Moreover, as the ripples in the VGC responses are also fixed, we can track variations in the power of this specific RF tone and correlate changes in power with signal degradation. We use the same pump and probe wavelengths (1551 nm and $1559 \mathrm{~nm}$, respectively) as described previously and measure the RF spectra of the 160 $\mathrm{GHz}$ pulse train at the output of the OMUX and after propagation through $50 \mathrm{~m}, 100 \mathrm{~m}$, and $150 \mathrm{~m}$ of SMF (corresponding to dispersions of $0.85 \mathrm{ps} / \mathrm{nm}, 1.7 \mathrm{ps} / \mathrm{nm}$, and 
$2.55 \mathrm{ps} / \mathrm{nm})$. The measurement conditions are the following: for operation in Channel \#1, the total power into the integrated device is fixed at $17.5 \mathrm{dBm}$, of which the probe accounts for 7 $\mathrm{dBm}$ while for operation in Channel \#2, the total power into the integrated device is fixed at $21 \mathrm{dBm}$, of which the probe accounts for $8 \mathrm{dBm}$.

The results are highlighted in Fig. 7 where the input (pump) signal is launched into Channel \#1, which shows that the XPM-induced optical spectra (and hence RF spectra) changes as the dispersion is varied (note that the VGC response contributes to the 'messy' spectra). Similar results are obtained when the input is launched into Channel \#2. We then focus on the $160 \mathrm{GHz}$ tone and measure the corresponding power. This power as a function of fiber length (dispersion) is summarized in Fig. 8. There is a clear reduction in tone power with increasing dispersion; this result is consistent with those reported by others (e.g., [9]).
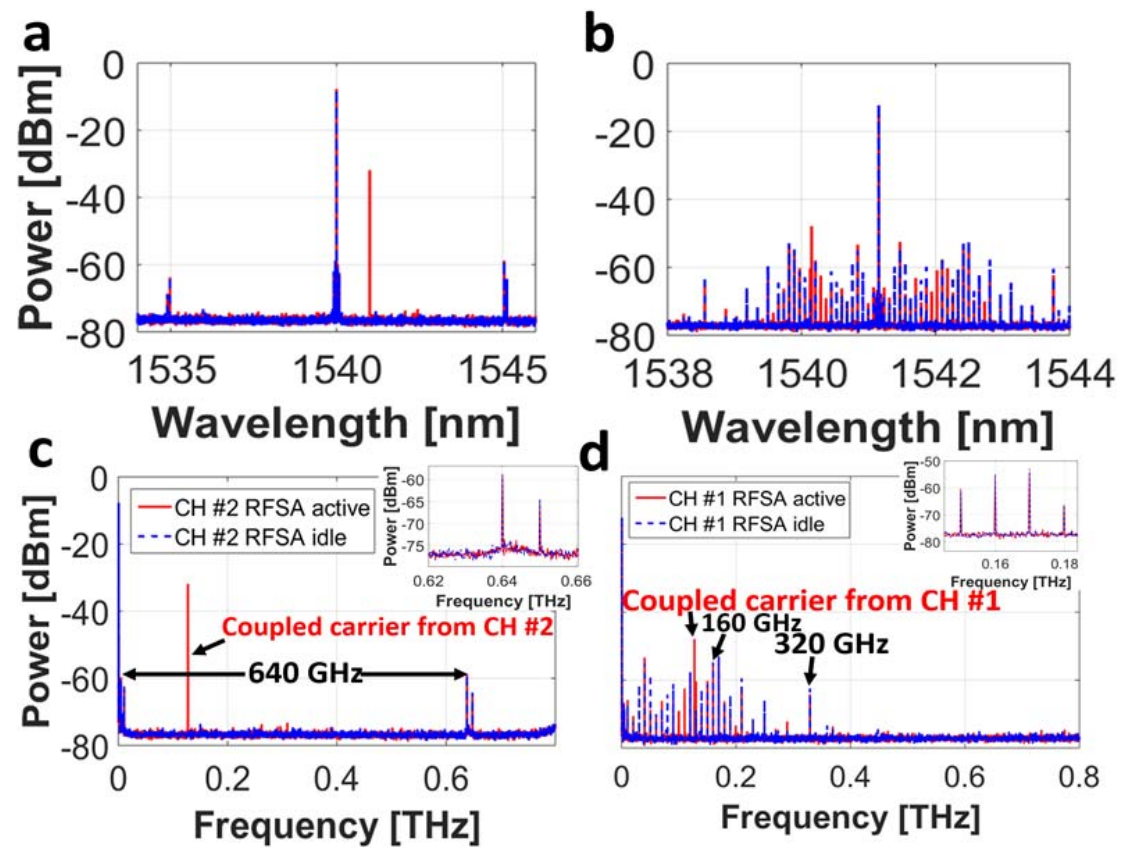

Fig. 6. Performance of simultaneous multi-channel RFSA when the probe carriers are offset by $1 \mathrm{~nm}$. XPM-induced sidebands are observed on (a) Channel \#1 and (b) Channel \#2. The half sideband RF spectrum are illustrated in (c) for Channel \#1 and (d) for Channel \#2. Enlarged spectra around $640 \mathrm{GHz}$ and $160 \mathrm{GHz}$ tones are shown in the insets. The resolution bandwidth of the OSA is $20 \mathrm{MHz}$.
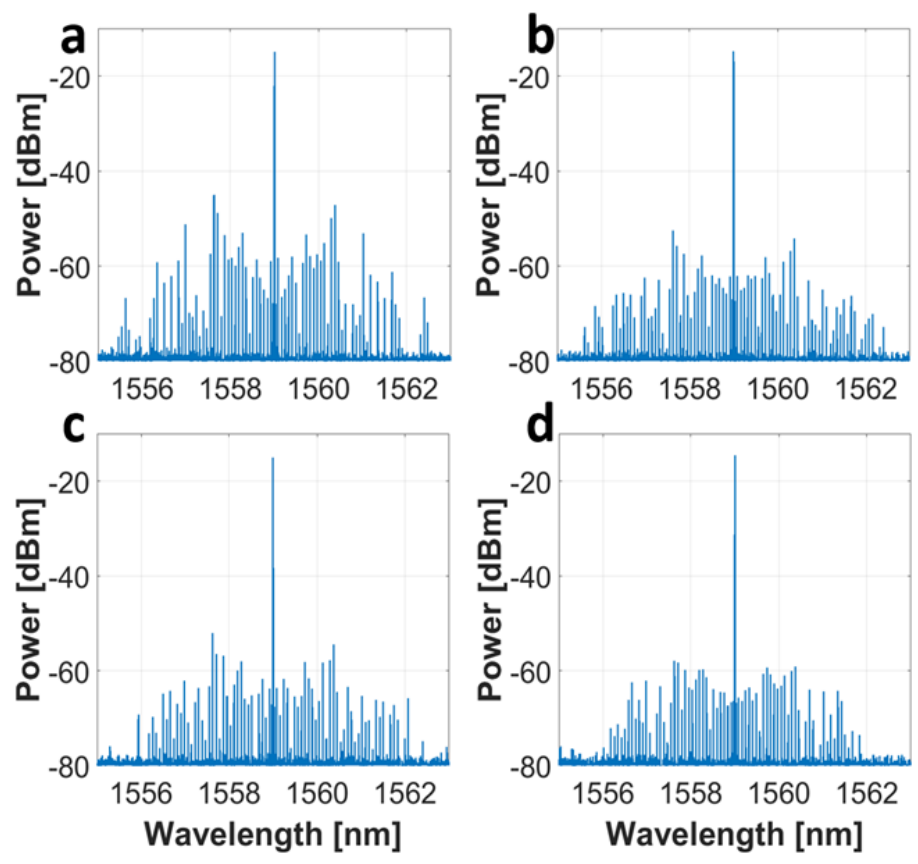

Fig. 7. Measured XPM-induced optical spectra centered about the probe wavelength $(1559 \mathrm{~nm})$ for the $160 \mathrm{GHz}$ pulse train launched into Channel \#1 after propagation through (a) no fiber, (b) $50 \mathrm{~m}$, (c) $100 \mathrm{~m}$, and (d) $150 \mathrm{~m}$ of SMF. 


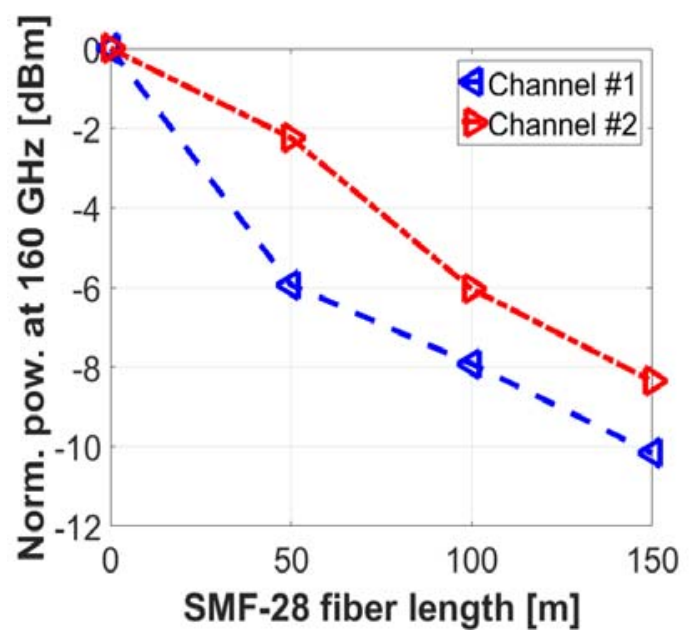

Fig. 8. Variation in power of the $\mathrm{RF}$ tone at $160 \mathrm{GHz}$ (obtained from the measured XPM-induced optical spectra) as a function of fiber length. The results are normalized to the RF tone power when the pulse train does not propagate through fiber.

\section{DISCUSSION}

We now discuss three aspects for improving the performance of multi-channel RFSA: (1) how to obtain similar operating bandwidth on multiple channels, (2) the impact of device coupling on overall RFSA operating bandwidth, and (3) how to further increase the number of operating channels. It is highly preferred to retain comparable bandwidth on each channel in the multi-channel RFSA operation. Since the modal profile on each channel in our proposed scheme is distinct, it needs careful design to optimize the dispersion profile on each channel. For instance, owing to greater dispersion, Channel \#2 (in $\mathrm{TE}_{1}$ mode) of the MSND has a BW that is less than a third of that on Channel \#1. By optimizing the dimension of our multi-mode waveguide, we may improve the BW on Channel $\# 2$ and even those of higher-order modes ( $\mathrm{TE}_{2}$ and above) so that each channel has a comparable bandwidth in RFSA. Fig. 9 is an example of simulated dispersion profiles for the first 6 TE modes $\left(\mathrm{TE}_{0}-\mathrm{TE}_{5}\right)$ in a multi-mode silicon waveguide with a cross-section of $4.5 \mu \mathrm{m}$ wide $\times 220 \mathrm{~nm}$ high. The absolute values of GVD in high-order TE modes become less than that in $\mathrm{TE}_{0}$ mode, which indicates that for the same signal-pump detuning, the bandwidth potential in high-order TE mode is even greater (e.g., The bandwidth in $\mathrm{TE}_{5}$ can be 4 times greater than in $\mathrm{TE}_{0}$ ). One the other hand, it may be useful for RFSA bandwidth improvement to replace the VGCs in the MSND with edge couplers [19]. Edge coupling itself imposes less bandwidth constraints and hence has little impact on the overall frequency response of device. With edge coupling, the RFSA bandwidth is then largely determined by the intrinsic bandwidth of the multi-mode waveguide. Furthermore, the coupling loss through edge coupler $(<1 \mathrm{~dB}$ over $80 \mathrm{~nm}$ in [19]) tends to be less than that of vertical coupling, thereby reducing the optical power required to launch into the MSND.

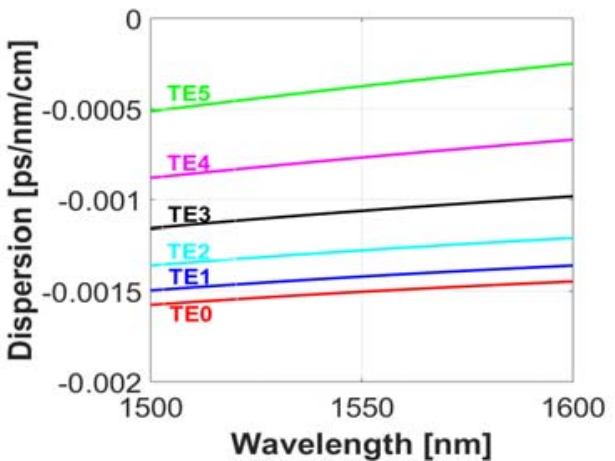

Fig. 9. Dispersions from $1500 \mathrm{~nm}$ to $1600 \mathrm{~nm}$ for $\mathrm{TE}_{0}-\mathrm{TE}_{5}$ modes in a $4.5 \mu \mathrm{m}$ $(W) \times 220 \mathrm{~nm}(H)$ multi-mode waveguide.

Secondly, the generation mechanism of high-order modes through ADC does not constrain the channel number in simultaneous operation either. Experimentally, Wang et al. has demonstrated exciting up to 8 modes in a multi-mode waveguide $\left(\mathrm{TE}_{0}-\mathrm{TE}_{3}\right.$ and $\left.\mathrm{TM}_{0}-\mathrm{TM}_{3}\right)$ using ADCs [20]. The mode (de-)MUX can be implemented using various structures; the advantage of the ADC is its relatively simple design. However, as the number of higher-order modes generated by the coupler is increased, careful design is necessary to maintain low linear cross-talk among the modes. The results shown in [20] are the first proof-of-concept for exciting several modes using an ADC and there is likely room to improve performance further.

We have simulated the effective areas for the different modes of a multi-mode silicon waveguide (same dimensions as before). As expected, the effective mode area decreases for higher-order modes and this will increase the input power requirements necessary to obtain a similar nonlinear phase shift compared to the lowest order mode [15]. Based on our nonlinear cross-talk characterization, we expect the nonlinear coefficient between modes to be even smaller for higher-order modes (e.g., between $\mathrm{TE}_{0}$ and $\mathrm{TE}_{2}$ ) due to increased walk-off arising from modal and group velocity dispersion.

We can envisage a scenario where a higher number of signal channels $(N>2)$ are under RFSA measurement. While all SUTs are launched into silicon integrated device at different input ports but in the same $\mathrm{TE}_{0}$ mode, each SUT is then routed onto an arbitrary channel of the device through the mode-conversion in ADCs. For each channel, another CW light wave accompanies the SUT for the photonic RFSA. Once the ultra-fast nonlinear XPM is accomplished, the phasemodulated $\mathrm{CW}$ light waves are all transformed back to $\mathrm{TE}_{0}$ mode again and coupled out of the device for further spectrum measurements. By exploiting polarization diversity, bidirectional propagation, and an appropriately designed $\mathrm{mm}$ NLWG, our approach to achieve simultaneous multi-channel RFSA can be scaled up to an even higher channel number.

Lastly, the design of mode-selective nonlinear device can be implemented using other nonlinear device platforms/materials, e.g., silicon nitride $[21,22]$ and chalcogenide waveguides [23]. For example, silicon nitride can offer advantages in small propagation loss (on the order of $0.03 \mathrm{~dB} / \mathrm{cm}$ [21]), relatively large nonlinearity, and negligible nonlinear loss. Chalcogenide waveguide intrinsically possesses a large Kerr nonlinear 
coefficient, thereby generating greater amount of nonlinearities than silicon with a reasonable input power.

\section{CONCLUSION}

In summary, we have demonstrated simultaneous multichannel RF spectrum analyzer for $640 \mathrm{GHz}$ and $160 \mathrm{GHz}$ optical waveforms in a single multi-mode waveguide. Furthermore, the effective 3-dB bandwidths of RFSA on both channels are determined to be $1.4 \mathrm{THz}$ and $0.5 \mathrm{THz}$, respectively. As our results match other reported ones [6, 9], the RFSA performance on each channel in our MSND is indeed robust in multi-channel operation. Moreover, in terms of RFSA bandwidth and high-order mode generation, we also analyze the possibility of simultaneous RFSA with a higher number of channel. We believe that the method of harnessing mode-selective excitation of nonlinear optical effects has much potential to scale to a higher number of channels and opens up a new degree of freedom in realizing multi-channel optical performance monitoring, photonic RF arbitrary waveform generation and optical source characterization.

\section{ACKNOWLEDGMENT}

The devices were fabricated by Richard Bojko at the University of Washington Nanofabrication Facility, a member of the National Science Foundation National Nanotechnology Infrastructure Network. We also thank Marwan Chehade and Muhammad Anees Mian for their contributions to the experimental work. Finally, we thank the reviewers for the useful feedback and comments.

\section{REFERENCES}

[1] D. C. Kilper, R. Bach, D. J. Blumenthal, D. Einstein, T. Landolsi, L. Ostar, M. Preiss, and A. E. Willner, "Optical performance monitoring," IEEE/OSA J. Lightw. Technol., vol. 22, no. 1, pp. 294-304, Jan. 2004.

[2] P. Samadi, L. R. Chen, C. Callender, P. Dumais, S. Jacob, and D. Celo, "RF arbitrary waveform generation using tunable planar lightwave circuits," Opt. Commun., vol. 284, no. 15, pp. 3737-3741, Jul. 2011.

[3] J. Schröder, T. D. Vo, and B. J. Eggleton, "Repetition-rate-selective, wavelength-tunable mode-locked laser at up to $640 \mathrm{GHz}$," Opt. Lett., vol. 34, no. 24, pp. 3902-3904, Dec. 2009.

[4] V. Torres-Company and L. R. Chen, "All-fiber joint-transform correlator for time-multiplexed signals," Opt. Lett., vol. 34, no. 21, pp. 3385-3387, Nov. 2009.

[5] C. Dorrer and D. N. Maywar, "RF spectrum analysis of optical signals using nonlinear optics," IEEE/OSA J. Lightw. Technol., vol. 22, no. 1, pp. 266-274, Jan. 2004.

[6] B. Corcoran, T. D. Vo, M. D. Pelusi, C. Monat, D.-X. Xu, A. Densmore, R. Ma, S. Janz, D. J. Moss, and B. J. Eggleton, "Silicon nanowire based radio-frequency spectrum analyzer," Opt. Express, vol. 18, no. 19, pp. 20190-20200, Sep. 2010.

[7] T. D. Vo, B. Corcoran, J. Schroder, M. D. Pelusi, D. X. Xu, A. Densmore, R. Ma, S. Janz, D. J. Moss, and B. J. Eggleton, "SiliconChip-Based Real-Time Dispersion Monitoring for $640 \mathrm{Gbit} / \mathrm{s}$ DPSK Signals," IEEE/OSA J. Lightw. Technol., vol. 29, no. 12, pp. 17901796, Jun. 2011.

[8] M. Ferrera, C. Reimer, A. Pasquazi, M. Peccianti, M. Clerici, L. Caspani, S. T. Chu, B. E. Little, R. Morandotti, and D. J. Moss, "CMOS compatible integrated all-optical radio frequency spectrum analyzer," Opt. Express, vol. 22, no. 18, pp. 21488-21498, Sep. 2014.

[9] M. Pelusi, F. Luan, T. D. Vo, M. R. E. Lamont, S. J. Madden, D. A. Bulla, D.-Y. Choi, B. Luther-Davies, and B. J. Eggleton, "Photonicchip-based radio-frequency spectrum analyser with terahertz bandwidth," Nat. Photon., vol. 3, no. 3, pp. 139-143, Mar. 2009.
[10] T. D. Vo, M. D. Pelusi, J. Schröder, F. Luan, S. J. Madden, D. Y. Choi, D. A. P. Bulla, B. Luther-Davies, and B. J. Eggleton, "Simultaneous multi-impairment monitoring of $640 \mathrm{~Gb} / \mathrm{s}$ signals using photonic chip based RF spectrum analyzer," Opt. Express, vol. 18, no. 4, pp. 39383945, Feb. 2010.

[11] H. G. Park, C. C. Pohalski, and B. Y. Kim, "Optical Kerr switch using elliptical-core two-mode fiber," Opt. Lett., vol. 13, no. 9, pp. 776-778, Sep. 1988.

[12] H. R. Stuart, "Dispersive multiplexing in multimode optical fiber," Science, vol. 289, no. 5477, pp. 281-283, Jul. 2000.

[13] D. J. Richardson, J. M. Fini, and L. E. Nelson, "Space-division multiplexing in optical fibres," Nat. Photon., vol. 7, no. 5, pp. 354-362, May 2013.

[14] Y. Ding, J. Xu, H. Ou, and C. Peucheret, "Mode-selective wavelength conversion based on four-wave mixing in a multimode silicon waveguide," Opt. Express, vol. 22, no. 1, pp. 127-135, Jan. 2014.

[15] M. Ma and L. R. Chen, "Harnessing mode-selective nonlinear optics for on-chip multi-channel all-optical signal processing," $A P L$ Photonics, vol. 1, no. 8, pp. 086104, Nov. 2016.

[16] Y. Ding, J. Xu, F. Da Ros, B. Huang, H. Ou, and C. Peucheret, "Onchip two-mode division multiplexing using tapered directional couplerbased mode multiplexer and demultiplexer," Opt. Express, vol. 21, no. 8, pp. 10376-10382, Apr. 2013.

[17] A. D. Bristow, N. Rotenberg, and H. M. van Driel, "Two-photon absorption and Kerr coefficients of silicon for 850-2200nm," Appl. Phys. Lett., vol. 90, no. 19, pp. 191104, May 2007.

[18] R. Adams, M. Spasojevic, M. Chagnon, M. Malekiha, J. Li, D. V. Plant, and L. R. Chen, "Wavelength conversion of 28 GBaud 16-QAM signals based on four-wave mixing in a silicon nanowire," Opt. Express, vol. 22, no. 4, pp. 4083-4090, Apr. 2014.

[19] B. B. Bakir, A. V. d. Gyves, R. Orobtchouk, P. Lyan, C. Porzier, A. Roman, and J. M. Fedeli, "Low-Loss $(<1 \mathrm{~dB})$ and polarizationinsensitive edge fiber couplers fabricated on 200-mm silicon-oninsulator wafers," IEEE Photonics Technol. Lett., vol. 22, no. 11, pp. 739-741, Feb. 2010.

[20] J. Wang, S. He, and D. Dai, "On-chip silicon 8-channel hybrid (de)multiplexer enabling simultaneous mode- and polarizationdivision-multiplexing," Laser Photonics Rev., vol. 8, no. 2, pp. L18L22, Mar. 2014.

[21] J. F. Bauters, M. J. R. Heck, D. John, D. Dai, M.-C. Tien, J. S. Barton, A. Leinse, R. G. Heideman, D. J. Blumenthal, and J. E. Bowers, "Ultralow-loss high-aspect-ratio Si3N4 waveguides," Opt. Express, vol. 19, no. 4, pp. 3163-3174, Feb. 2011

[22] D. J. Moss, R. Morandotti, A. L. Gaeta, and M. Lipson, "New CMOScompatible platforms based on silicon nitride and Hydex for nonlinear optics," Nat. Photon., vol. 7, no. 8, pp. 597-607, Aug. 2013.

[23] B. J. Eggleton, B. Luther-Davies, and K. Richardson, "Chalcogenide photonics," Nat. Photon., vol. 5, no. 3, pp. 141-148, Mar. 2011.

Ming Ma (S'11) received the B.Eng. in electrical engineering from Beijing Institute of Technology, Beijing, China and the M.Sc. degree in electrical engineering from Technical University of Denmark, Denmark and is currently working toward the Ph.D. degree in optical communication systems with the Photonics System Group, McGill University, Montreal, QC, Canada.

Rhys Adams received the B.Eng. and M.Eng. degrees in electrical and computer engineering from McGill University, Montreal, QC, Canada, in 2002 and 2005 respectively. He has been with the Department of Physics at Vanier College since 2006. His research interests include optical communications, microwave photonics, and physics education and pedagogy.

Lawrence R. Chen (S'95-SM'05) received the B.Eng. degree in electrical engineering and mathematics from McGill University, Montreal, QC, Canada, in 1995 and the M.A.Sc. and Ph.D. degrees in electrical and computer engineering in 1997 and 2000, respectively, from the University of Toronto, Toronto, ON, Canada. Since 2000, he has been with the Department of Electrical and Computer Engineering at McGill University. His research interests are in optical communications, fiber and integrated optics, and microwave photonics (in particular, active and passive devices in silicon photonics for optical and microwave signal processing) as well as engineering education and teaching pedagogy.

Dr. Chen is an Editor for Optics Communications and an Associate Editor for IET Electronics Letters. 